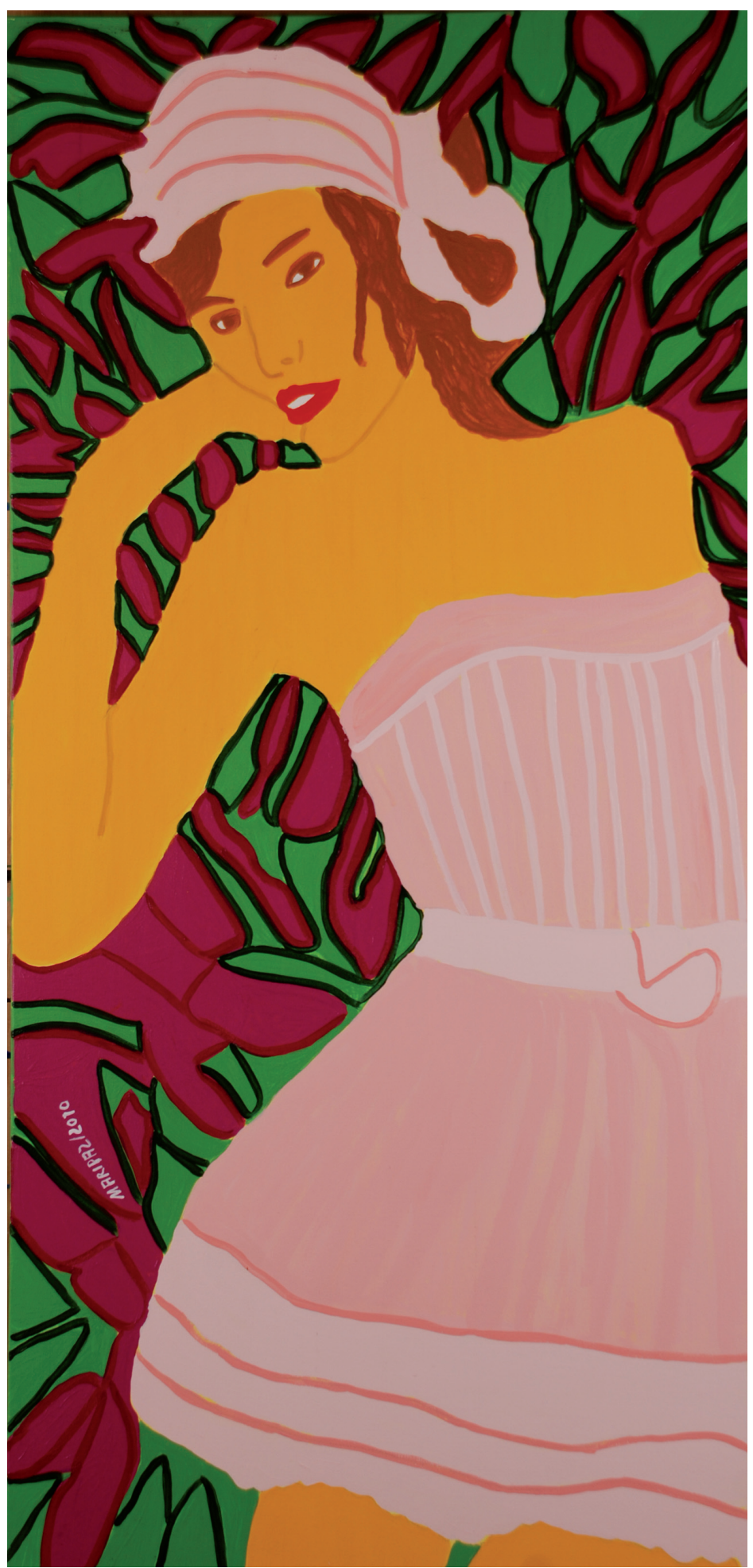

Serie Ellas. Ellas 8

Acrílico sobre lienzo. 2010. 1,20 x 0,60 mts.

Maripaz Jaramillo 


\section{Ir a cine, ver cine, escribir sobre cine La crítica de cine en Colombia}

Fecha de recepción: 19 de enero de 2011 - Aprobación: 23 de marzo de 2011

\section{Juan Gustavo Совo Borda}

\section{Resumen}

En este escrito, el autor hace un recorrido por la historia de la crítica de cine en Colombia y su evolución. Con minuciosidad, relata cómo se ha entendido este oficio a través de los años y presenta la historia de los críticos cinematográficos más importantes del país.

\section{Abstract}

This is a journey that the author makes throughout the history of film criticism in Colombia and its evolution. It tells, with great attention to detail, how this trade has been understood through the years and it also presents the history of the most important film critics in the country.

\section{Palabras clave}

Cine, ver cine, escribir sobre cine, crítica de cine en Colombia

\section{Keywords}

Movies, watch movies, write about film, film criticism in Colombia
Los hermanos italianos Francisco y Vicente di Domenico llegan al país en 1911. Ya en 1919 tenían en Barranquilla el Teatro Colombia con capacidad para 5.500 espectadores donde además del cine, su escenario podía adaptarse a compañías de danza, drama, opereta y zarzuela y la platea transformarse "en amplio circo para toros y circos ecuestres”, además de contar con amplios salones para reuniones, bailes y soirées.

Así lo anunciaban orgullosos en su revista Películas cuyo número 122, fechada en mayo de 1919, era dirigido por Francisco
Bruno. En esa edición se entrevista a Vicente di Domenico rodeado de fotos de actrices italianas como Francisca Bertini en pose trágica. Comprar una película de esta diva como Cuatro pecados le habría costado 20.886 liras. Las entradas por una noche en Bogotá ascienden desde 580 dólares a 1. 036. Al salir de Italia, hace 12 años, este admirador de "los nobles reyes de Italia", tenía un capital de 25.000 liras y tras vender telas y bisuterías descubrió más tarde la veta del cinematógrafo. Es la época en que 25 millones de espectadores en el mundo entero en 100.000 teatros dejan en 
las cajas de los cines 50 millones de francos por día. Ahora esta sociedad familiar de seis socios, proveniente de Salerno, de un pueblecito llamado Castelnuovo di Conza, distribuye sus películas de Chiquinquirá a Cartago y de Tumaco a Pereira.

Paralelamente, en ese ejercicio publica en su revista poemas de Leopoldo Lugones,

El mundo había cambiado, el cine, crecido, pero Colombia, al parecer, seguía alimentándose de productos extranjeros; en este caso, de los filmes norteamericanos y las películas mexicanas.
Alfonsina Storni y Nicolás Bayona Posada más las prosas de Luis Tejada, Armando Solano ("Maître Renard") y Baldomero Sanín Cano. El presidente Pedro Nel Ospina les solicitaba ver nuevas películas y allí acudían, a Palacio, de carrera, con los equipos para ofrecerlas a grandes y chicos.

Registraban en esa revista las disputas bélicas por el Fiume y sacaban avisos de página entero de su compatriota Tito Ricci quien en mármol de carrara había erigido un monumento de 13 metros de altura colocado en la Plaza de los Mártires de Tunja.

\section{Reseña de autor \\ Juan Gustavo Cobo Borda (Colombia) \\ www.coboborda.org \\ coborda@gmail.com \\ Colaborador Revista Poliantea}

Poeta y ensayista bogotano. Fue director durante una década (1973-1984) de la revista Eco, de la librería Buchholz, y Gaceta, del Instituto Colombiano de Cultura. Ha ocupado cargos diplomáticos en Buenos Aires y Madrid y fue embajador en Grecia. Miembro número de la Academia Colombiana de la Lengua desde 1993, y correspondiente, de la Academia Española. Ha sido jurado tres veces del Premio Juan Rulfo, (Guadalajara, México); del Rómulo Gallegos, (Caracas); del Reina Sofía de poesía iberoamericana (Madrid) del Neustad, Universidad de Oklahoma, Estados Unidos y del Pablo Neruda (Chile).

Entre sus poemarios figuran La musa inclemente (2001) y La Patria boba (2008). Algunas de sus publicaciones son Lengua Erótica: antología poética para hacer el amor (Villegas Editores, 2004), Lector impenitente, El olvidado arte de leer, Enclave de palabras número tres Acosado animal (Politécnico Grancolombiano, 2010), Vargas Llosa: la pasión de narrar (Alfaguara, 2010) y su breviario arbitrario de la literatura colombiana (2011).
Emprendedores, infatigables, Vicente di Doménico haría en 1923 Aura o las violetas, una de las primeras películas colombianas. Aquello que las casas Lumiêre o Pathé habían iniciado desde Paris, enviando fotógrafos ambulantes por todo el mundo, daba frutos en la remota Colombia.

Vicente terminaría en Barcelona (España), pidiendo noticias sobre Colombia y Francisco como hotelero en Villeta. El trópico absorbió así su energía empresarial y su visión futurista de los negocios. La revista Películas que aún seguía publicándose en 1922 por sus artículos, biografías de actores y actrices, y fotografías del "arte cinematográfico y teatral" es indudable pionera de lo que pudiera considerarse una crítica cinematográfica traducida, aclimatándose poco a poco en Colombia. Pero lo relevante es volver a disfrutar la emoción de esos avisos promocionales al hablar de esa "monumental serie de Pathé" y suscitar la curiosidad con afirmaciones como "Los episodios de mayor sensación. Puede decirse que en esta serie no hay un instante en que el público no esté intrigado por la notable trama y el misterioso desarrollo". Como quien dice: cine en estado puro.

\section{El trasegar de la imagen}

Cuando Guillermo Cabrera Infante, el gran novelista cubano, ejercía de crítico cinematográfico en La Habana, en las hoy legendarias revistas Bohemia y Carteles, con el seudónimo de G. Caín, se despidió del oficio con un adiós plasmado en un libro: Un oficio del siglo XX, donde reunía su trabajo de 1954 a 1960. Su irónica y melancólica página final consignaba un agradecimiento: "A los desconocidos amigos de Guión de Bogotá, un apretón de manos y un murmullo ininteligi- 
ble y agradecido por la apología, que parece un estirado pésame".

El mundo había cambiado, el cine, crecido, pero Colombia, al parecer, seguía alimentándose de productos extranjeros; en este caso, de los filmes norteamericanos y las películas mexicanas. Así lo atestigua un singular periódico-revista llamado Cine noticias que en su año II y dirigido por Alberto Rojas Gaona, aseguraba en la década de 1950 un tiraje de 10.000 ejemplares. Publicaba fotos sugestivas de María Félix y Martín Carol y ofrecía información sobre el mundo del cine en el exterior (Judy Garland) o el latinoamericano, donde Jorge Negrete interpretaba a Marcos Vargas, personaje de Rómulo Gallegos en su novela Canaima llevada al cine o bien, Libertad Lamarque era la mujer equis en la película del mismo nombre.

La llegada de Eastman Color y las semanas de homenaje al cine mexicano donde en 12 ciudades y 20 teatros se exhibirían en forma simultánea películas como Escuela de vagabundos con Pedro Infante y Miroslava o Un extraño en la escalera con Silvia Pinal y Arturo de Córdova, además del Robinson Crusoe de Luis Buñuel, realizado en México, su nueva tierra de adopción, comprueban que de las películas italianas de los Di Domenico habíamos pasado a las mexicanas, promovidas por distribuidores y embajadas. Y del invento del Pathé al Cinemascope, como se anuncia en esta ocasión: en el Teatro de la Comedia la primera producción en Cinemascope de la Universal filmada en technicolor, Atila frente a Roma con Charlton Helston y Lumille Sherina.

Vistavisión con Los diez mandamientos de Cecil B. de Mille y por su parte, Warner pagaba su aviso de El cáliz de plata con Pier Angeli, Jack Palance y "su gran debut": Paul Newman.

El cine venia de Hollywood, lo comercializaban las transnacionales y estas publicaciones reproducían de forma literal sus comunicados de prensa, los desde siempre sabrosos chismes de romances y rupturas y las atractivas fotos de las estrellas, facilitadas por las casas distribuidoras. La farándula, en fin. Pero el cine colombiano no terminaba de aparecer. El editorial de agosto 27 de 1955 al hablar de la presentación de una nueva película nacional que no menciona dirá: "Ninguna película, ya sea bogotana, caleña o antioqueña ha tenido el aplauso del público. Es cierto que los teatros se han colmado de bote en bote... pero al público colombiano no podemos seguirlo estafando con "cosas" que dicen ser una película y no dejan de ser sino unas "mamarrachadas" del peor gusto".

La filmación en Cartagena y Barranquilla de escenas de Llama al viento basada en la novela de Alfonso López Michelsen; Los elegidos en producción mexicana con la actriz Adriana Walter que en 1984 tanto director y actores rusos la volverían a llevar a la pantalla con el aporte colombiano de Amparo Grisales. De otro lado, bajo este panorama, los 350000 dólares que ganara Cantinflas por La vuelta al mundo en 80 días cierra este recuento de Cine Noticias donde el pelado del habla popular enrevesada de las clases bajas de Ciudad de México se convierte en el criado del flemático caballero inglés que honra una apuesta de club. Ahora se llamará Passepartout: un comodín.

Con una óptica como esta, los asistentes a los teatros Faenza, Imperio, Americano, 
Aladino, Coliseo, Comedia, El Cid, Mogador, San Carlos, San Jorge, Metro o Teusaquillo -las salas tradicionales de la época en Bogotá-, aceptarán la versión comercial y edulcorada de un representante decisivo de la cultura popular latinoamericana, en su versión norteamericana para exportar.

El negocio del cine, hecho de arte y técnica, de innovación y comercialización, continuaba su marcha. Y a su lado, siempre, la tradición de la nota de prensa o la redacción del comentario hecho en Colombia.

De ahí que la verdadera aparición de la crítica de cine coincide con aquellos años cuando Jorge Gaitán Durán la inicia en El Espectador y al irse a Europa se la deja a Gabriel García Márquez quien la ejerce de febrero de 1954 a julio de 1955 en dicho diario. Y cuando Gaitán Durán y Hernando Valencia Goelkel fundan Mito (1955-1962) y Valencia Goelkel critica y analiza películas en Cromos. Ese es el auténtico punto de partida de la crítica de cine en Colombia.

\section{El Mito del cine en Mito}

Los cuarenta y dos números de Mito, publicados entre 1955 y 1962, fueron indudables pioneros en la historia de la crítica de cine en Colombia. Fiel a su tradición cosmopolita, Mito miró al mundo y prestó atención inteligente a este nuevo arte. Destaco, en primer lugar, el homenaje a Chaplin en su número cuatro, donde Jorge Gaitán Durán escribe sobre Tiempos Modernos, Hernando Salcedo sobre Monsieur Verdoux y Candilejas y Antonio Montaña repasa toda su trayectoria. Luego, su interés por el neorrealismo italiano y la nouvelle vague francesa, con su punto más alto en el valioso ensayo de Hernando Valencia Goelkel sobre
Hiroshima, mon amour de Alain Resnais, con guión de Marguerite Duras. Pero no se trata sólo de eso: su director, Jorge Gaitán Durán, viajero por Europa, reseñaba La Strada de Fellini; Francisco Norden, desde París, hablaba de René Clair; y Guillermo Angulo, después de estudiar en Cinecittà, se preocupaba por traer a Colombia lo que significaba Los 400 golpes de Truffaut, y el conocimiento del gran guionista del neorrealismo, Cesare Zavattini, de quien tradujo un delicioso cuento: «Cine». Gretel Wernher, por su parte, se internará en los Secretos de mujeres de Ingmar Bergman.

Acompañándolos, en varias ocasiones «el padre» Hernando Salcedo Silva reseñará los libros más recomendables para el estudio del cine, como lo eran la historia de Georges Sadoul y el clásico de Kracauer, De Caligari a Hitler, en torno al expresionismo alemán. Sin olvidar en ningún momento su devoción por el musical, las películas del oeste y de gánsteres, en su inclinación por el cine norteamericano, como lo atestiguan sus notas sobre El hombre del oeste $y$ Horizontes de grandeza, de Anthony Mann y William Wyler, con los legendarios Gary Cooper y Gregory Peck, respectivamente. Devoción en la cual lo acompañaría Hernando Valencia Goelkel, entusiasmado con Shane, de George Stevens. También vale la pena señalar que en 1955 Hernando Salcedo Silva comenta el Robinson Crusoe, de Luis Buñuel. Tendremos así un panorama variado del cine de entonces, en una revista no dedicada específicamente al cine sino a la cultura en general. A esto se añade que el primer director del Cine Club Colombiano sería Luis Vicens, un catalán importador de libros muy unido a Mito, y luego promotor de cine en México. 
Mito, dio así su nombre a una generación que también iba, hablaba y escribía de cine y que también dio batallas contra la censura cinematográfica del momento, que prohibió, en 1958, la exhibición de Rojo y Negro, basada en la novela de Stendhal. El Siglo apoyó la censura al decir que la novela estaba en el Índex y que la película había sido financiada por los comunistas. Gaitán Durán dijo al respecto: «Colombia es un país que ha escogido la inmovilidad». Y vio encarnar en ciertos ídolos personales sus sueños y aspiraciones. Tal la hermosa nota que Jorge Gaitán Durán escribió en El Espectador sobre Gerard Philippe, a raíz de su muerte, el 20 de diciembre de 1959: «Fue de cierto modo todo lo que nosotros hemos querido ser; nuestro esfuerzo desesperado por superar la corrupción y el terror de la vida moderna [...]. Nos impresiona que esta pulcritud, esta fogosidad, esta ambición, este esplendor, hayan sido vertiginosamente aniquilados por la absoluta miseria humana que es el cáncer».

¿No estaría la verdadera modernidad de Mito en haber hablado al tiempo de Hollywood y Visconti, de Cesare Zavattini y de Brecht y el cine, en haber traducido los diarios de filmación de Fellini en Las Romanas y en señalar cómo la forma de hablar de Cantinflas era la imagen más fiel de la oratoria política colombiana? Tal el acierto de Mito al referirse al cine.

"La revista Mito, de Bogotá, una de las mejores que haya tenido América Latina": así escribía el profesor francés Jacques Gilard en 1974, en el prólogo a El coronel no tiene quien le escriba en la edición colombiana del Círculo de Lectores (p. VI).

Allí también mostraba el influjo que en la narrativa de García Márquez había tenido su trabajo de comentarista cinematográfico en El Espectador y que ya se podía fechar desde el 16 de octubre de 1950 cuando reseña en El Heraldo de Barranquilla Ladrón de bicicletas de Vittorio de Sica.

En febrero de 1954 inaugurará en Bogotá su columna de crítica cinematográfica con un marcado énfasis admirativo por el neorrealismo italiano. Milagro en Milán, Indiscreción de una esposa, El abrigo, Alemania año cero y Umberto D son algunas de las películas que comenta, y elogia. Historias de gente común y corriente, de gente pobre, realizadas con técnicas elementales y directas, que contribuyen a perfilar lo humano en sus personajes, tal como sucedería en El Coronel, terminado en Paris en enero de 1957.

Al grupo que fue llamado, tal vez irónicamente, la generación de los maestros (King, 1994), podía adscribirse, sin problemas, García Márquez. Es así como Guillermo Angulo, Jorge Pinto, Álvaro González y Francisco Norden eran, en alguna forma sus compañeros generacionales, habían viajado a Europa y estudiado en el IDHEC en París o en el Centro Spirimetale en Roma, como fue el caso de Angulo y García Márquez. Pero en realidad el primer crítico cinematográfico colombiano, proveniente también de la revista Mito, fue Hernando Valencia Goelkel.

Lo dilatado de su cultura, atenta al ámbito anglo-sajón y a una revista como The New Yorker y Pauline Kael, lo punzante de su prosa y lo original de sus análisis, trátese de Bergman como de Antonioni, hacen de la lectura de Crónicas de cine (1974) un primer hito ineludible.

Cualquiera interesado en ese ir y venir entre lo nacional y lo extranjero tendrá tam-
El negocio del cine, hecho de arte y técnica, de innovación y comercialización, continuaba su marcha. Y a sulado, siempre, la tradición de la nota de prensa - la redacción del comentario hecho en Colombia. 
bién otros volúmenes de calidad disponibles. Me refiero a Crónicas del cine colombiano, 1897 - 1950 (1981) de Hernando Salcedo Silva o la Historia del cine colombiano (1978) de Hernando Martínez Pardo. También son útiles los tres volúmenes de Páginas de cine, de Luis Alberto Álvarez, publicados en 1988, 1992 y 1998 y varias veces reeditados. A ellos debe añadirse, sin lugar a dudas, la recopilación de Andrés Caicedo: Ojo al cine (2009), Otros libros como los Enfoques de película (2008) de Mauricio Laurens o de Orlando Mora amplían las páginas sobre la crítica de cine en Colombia.

Esta antología, al reconocer la importancia precursora de dichos aportes, busca ahora rescatar del limbo de las hemerotecas, piezas pioneras del análisis cinematográfico, de Clímaco Soto Borda y el poeta Guillermo Valencia, de Tomás Carrasquilla y Luis Tejada, en una proseguida tradición de narradores atentos a la importancia del cine, trátese de Hernando Téllez o de poetas como Jorge Gaitán Durán y Gustavo Ibarra, para llegar en las últimas generaciones a los ya fallecidos R.H. Moreno Durán y Alberto Duque López y a Alberto Sierra, narrador y promotor del cine club en Cartagena.

Al rescatar con Ramiro Arbeláez, desde Cali, y Cobo Borda, desde Bogotá, muchas de estas piezas, se contribuye al mapa de la pasión por el cine en Colombia. La pesquisa, en frágiles revistas que en muchos casos no alcanzaron más de dos o tres números, mostraba la garra del escritor fascinado por el espectáculo cinematográfico. Ansiosos de comunicar sus hallazgos, de compartir preguntas, dudas y evidencias. Trátese de los clásicos, caso de Buñuel visto desde tan variadas ópticas, trátese del compañero obsesionado no sólo por escribir sobre cine sino también hacerlo. Así sucedió en Cali, donde precisamente Ramiro Arbeláez, con Carlos Mayolo, Luis Ospina, Andrés Caicedo, Hernando Guerrero, Humberto Valverde y Sandro Romero Rey crearon una personal forma de enfocar el cine, con sus mitologías inconfundibles reflejadas en el Cine Club de Cali y la revista Ojo al cine. Igual sucedería en Antioquia con Víctor Gaviria y el Cine Club del Colombo Americano y la revista Kinetoscopio, la de más larga andadura en nuestro medio. Pero que tuvo su taller inicial en torno al cine en publicaciones como la Revista de la Universidad de Antioquia.

La revisión de la Revista de la Universidad de Antioquia, desde diciembre de 1985 a diciembre de 2008, y que abarca del número 202 al 294, da un saldo muy favorable en torno a la crítica de cine. Dirigida por escritores y periodistas dedicados a la actividad literaria, como es el caso de Juan José Hoyos, Héctor Abad Faciolince y Elkin Restrepo, su interés por el arte cinematográfico combina bien la mirada sobre figuras claves ya de la historia del cine, de Harold Loyd a Kurosawa, de Humphrey Bogart a Fellini y Antonioni, con la atención a un cine que surgía allí mismo en Medellín, y que encarna un poeta amigo de los directores de la revista, como Víctor Gaviria, y su película Rodrigo D: no futuro (1990). En tal sentido las contribuciones de Juan Carlos González, a partir del año 2001, forman un breviario informado del cine contemporáneo y de los maestros consagrados, como lo atestigua también el libro que escribió sobre Francois Truffaut, Una vida hecha cine (Panamericana).

Adicionalmente, Pedro Adrián Zuluaga ha recobrado el clima que se vivía, y sus antecedentes más conspicuos. Paul Bardwell como director del Colombo-Americano 
y la legendaria revista Kinetoscopio, aparecida en 1990 y que aún sigue activa, y el padre Luis Alberto Álvarez, que llegado de Europa, hacía proselitismo en el GoetheInstitut a favor de Herzog y Faasbinder, y publicaba en El Colombiano los muy perspicaces ensayos y notas que perduran en los tres volúmenes de Páginas de cine que editaría precisamente la Universidad de Antioquia, a partir de 1988 y para concluir en 1998, son los otros puntos de referencia, en este proselitismo crítico e informativo sobre el cine, de innegable importancia.

Pero al margen de la fugacidad de los recortes de periódico y la precariedad absoluta de las revistas, muchos colombianos han añadido a la visión singular del cineasta la suya propia, o han reconocido, así mismo, cómo los sucesivos inicios del cine colombiano, que despunta y agoniza cíclicamente, mucho nos dice sobre nosotros mismos, sobre el campo y la ciudad, sobre la historia y la política, sobre la gente, sus frustraciones, sueños y sentimientos. Proimagenes en Movimiento, que tanto ha contribuido a hacer cine, piensa en sus trece años de existencia que bien vale la pena adensar el horizonte de nuestra cultura, con el rescate de estas válidas y curiosas páginas sobre la crítica de cine en Colombia.

\section{Bibliografía Sugerida}

1. King, John. (1994). El carrete mágico. Una historia del cine latinoamericano.

2. Osorio, Oswaldo. (2008). Historiografía del cine colombiano. La saga atrasada de un cine que camina lento, en Cine colombiano. Investigación e historiografía. Bogotá, Cuadernos de Cine Colombiano.

3. Publicaciones periódicas de cine y video en Colombia, 1908 - 2007. Fundación Patrimonio Fílmico Colombiano. 